\title{
Quantitative determination of glycopyrrolate in human plasma by liquid chromatography-electrospray ionization mass spectrometry: The use of a volatile ion-pairing agent during both liquid-liquid extraction and liquid chromatography
}

\author{
M.L. Storme ${ }^{\mathrm{a}}$, R.S. t'Kindt ${ }^{\mathrm{a}}$, W. Goeteyn ${ }^{\mathrm{a}}$, K. Reyntjens ${ }^{\mathrm{b}}$, J.F. Van Bocxlaer ${ }^{\mathrm{a}, *}$ \\ a Laboratory of Medical Biochemistry and Clinical Analysis, Faculty of Pharmaceutical Sciences, Ghent University, Harelbekestraat 72, B-9000 Ghent, Belgium \\ ${ }^{\mathrm{b}}$ Department of Anaesthesia, Ghent University Hospital, De Pintelaan 185, B-9000 Ghent, Belgium
}

\section{A R T I C L E I N F O}

\section{Article history:}

Received 30 November 2007

Accepted 8 October 2008

Available online 14 October 2008

\section{Keywords:}

LC-MS/MS

Glycopyrrolate

Pharmacokinetics

\begin{abstract}
A B S T R A C T
The work presented here deals with the development of a quantitative tool for the determination of the quaternary ammonium anticholinergic glycopyrrolate in human plasma samples. Mepenzolate was used as an internal standard. The plasma samples were subjected to a suitable sample clean-up consisting of a simple and relatively fast, two step liquid-liquid ion-pair extraction procedure. The chromatography, using the same volatile ion-pair reagent heptafluorobutyric acid (HFBA), takes only 10 min. Relative standard deviation of retention times was never above $2.26 \%(n=36)$. The method was fully validated based on the US FDA Bioanalytical Method Validation Guidance for Industry. As such, a quantitative ESI-LC-MS(/MS) (TOF mass spectrometry) method was optimized for the absolute quantification of glycopyrrolate in human plasma in a concentration range from 0.101 to $101 \mathrm{ng} / \mathrm{mL}$ using a quadratic calibration function $\left(R^{2}=0.9995\right), y=-2.21 \times 10^{-4}\left( \pm 3.93 \times 10^{-5}\right) \times x^{2}+5.85 \times 10^{-2}$ $\left( \pm 5.27 \times 10^{-3}\right) \times x+4.08 \times 10^{-3}\left( \pm 4.82 \times 10^{-4}\right)$. For the three $\mathrm{QC}$ concentrations $\left(\mathrm{QC}_{1} 0.252, \mathrm{QC}_{2} 2.52\right.$, and $\left.\mathrm{QC}_{3} 25.2 \mathrm{ng} / \mathrm{mL}\right)$ and the LLOQ $(0.101 \mathrm{ng} / \mathrm{mL})$, total precision was under $20 \%(18.0 \%(n=6)$ at the LLOQ) and maximum accuracy was $112 \%$ ( $88.9 \%$ for the LLOQ $n=6$ ). Absolute matrix effect (maximum $133 \% \pm 9.59$, $n=3$ ), absolute recovery (better than $41.8 \% \pm 2.22, n=3$ ), relative (inter-subject) matrix effect (maximum $10.9 \% \pm 1.45, n=4$ ) and process efficiency (better than $45.2 \% \pm 5.74, n=3$ ) too were assessed at the $3 \mathrm{QC}$ concentrations.
\end{abstract}

(C) 2008 Elsevier B.V. All rights reserved.

\section{Introduction}

Quaternary ammonium (QA) drugs are widely used as anticholinergic agents. Glycopyrrolate (GLY), as a synthetic QA compound, has been used for decades as a pre-operative antimuscarinic. In addition, it diminishes the volume and free acidity of gastric secretions and controls excessive pharyngeal, tracheal, and bronchial secretions [1-4]. In contrast to the tertiary amines atropine sulfate and hyoscine hydrobromide, which are other well-known anticholinergic agents, the highly polar quaternary ammonium group of GLY limits its passage through the blood-brain barrier. As a consequence, doses which cause these marked antisialagogue actions have little effect on heart rate or pupil size. Intraoperatively, GLY counteracts drug-induced or vagal traction reflexes with the associated arrhythmias and oral and gastric secre-

\footnotetext{
* Corresponding author. Tel.: +32 9264 8131; fax: +329264 8197 .

E-mail address: Jan.VanBocxlaer@ugent.be (J.F. Van Bocxlaer).
}

tions. Although many anesthesiologists still routinely make use of GLY because of the positive effects, its use has been cut back during the last 20 years, due to the need for painful and anxiety-provoking intramuscular (IM) injection caused by short distribution and elimination half-life [5]. Clifford et al. investigated other administration routes, such as oral and intravenous (IV) routes. As expected by the known variable gastrointestinal absorption of GLY, oral administration was found to be ineffective. IV administration on the other hand proved to be superior to IM administration because of an additional decrease of gastric secretions, not seen by IM administration [1].

The scope of this study, as part of a larger clinical study, was to develop an analysis method for GLY, allowing a pharmacokinetic evaluation of patients treated with Robinul ${ }^{\circledR}$ after IV distribution through an arterial catheter. Past PK studies in normal volunteers, given a single IV infusion of $0.4 \mathrm{mg}$ GLY, already indicated that the drug undergoes a rapid distribution/elimination phase $\left(t_{1 / 2}=1.7 \mathrm{~h}\right)$. The peak plasma concentrations occur within the minute after infusion and no wide distribution to the tissues takes place [6]. As for all anticholinergics, the duration of action varies, with possi- 
ble antisialagogue effects for up to $7 \mathrm{~h}$. Each Robinul ${ }^{\circledR}$ injection vial contains $1 \mathrm{~mL}$ of $0.4 \mathrm{mg}$ GLY, USP in water for injection with $9 \mathrm{mg}$ of benzyl alcohol as preservative (Information for health professionals, Robinul data sheet). For IM injection, the onset of reaction is 20-40 min with peak plasma concentrations after approximately 30-45 min and the duration of action ranging from 4 to $6 \mathrm{~h}$.

During the past decades, several studies investigated possible quantitation approaches for GLY and other QA compounds. Due to their cationic character, detection of the latter drugs is still not straightforward. Coverage with immunoassays is limited. Traditionally, GLY was analyzed by using enzyme-linked immunosorbent assay (ELISA) [7,8]. However, cross-reactions are often noted. Methods using gas chromatography-mass spectrometry (GC-MS) on selected QA drugs have been developed but intact QA compounds cannot be analyzed with GC-MS [9-12]. One approach is the hydrolysis of the isolated quaternary compound to cyclopentylmandelic acid which is then amenable to detection and confirmation following derivatization and analysis by GC-MS. A broad range of other chromatographic and spectrometric techniques have been reported for the analysis of $\mathrm{QA}$ drugs [13-26]. An inherent disadvantage of most of these analytical methods is the lack of specificity, resulting in identification and quantification issues in complex matrices, e.g., plasma [13]. Only methods using mass spectrometry can unmistakably identify the detected analyte. Consequently, more specific methods like capillary electrophoresis-mass spectrometry (CE-MS) have been optimized for the analysis of QA, however, only in (horse) urine [9]. For QA drugs, indeed, CE seems a better option considering the permanent ionic characteristic of the analyte molecules. Unfortunately, coupling CE to MS is far from routine and does lack some robustness.

Liquid chromatography mass spectrometry (LC-MS) at the other hand, using electrospray ionization(ESI), has become the method of choice in both quantitative and qualitative bioanalytical work due to its speed, sensitivity, specificity and robustness [13]. In reversed phase type of separations, ion-pair chromatography allows the chromatography of ionic analytes. Generally, ion-pair chromatography is performed with high concentrations of non-volatile ion-pair reagents, obviously incompatible with (ESI-)mass spectrometry. Some of these issues can be overcome by phase-system switching using an ion-exchange trapping column (2D-LC) but, additional equipment $[17,27]$ is required and the complexity of the analytical method is substantially enhanced. The use of volatile acids as ionpairing agents was first described by Castro et al. [12], allowing the direct coupling of ion-pair chromatography and mass spectrometry. Heptafluorobutyric acid (HFBA), pentafluoropropionic acid (PFPA) and trifluoroacetic acid (TFA) were selected as possible ion-pairing agents. In their setup, HFBA yielded the most promising results. Using the latter approach, Ariffin and Anderson [28] developed an efficient procedure for the simultaneous determination of QA drugs and herbicides in human whole blood using SPE extraction.

Solid phase extraction, mainly based upon cation-exchange, is the most investigated extraction method for QA compounds [29]. Indeed, the cationic nature of the drugs does not lend itself well to liquid-liquid partition extraction methods [6,9,12-26]. Liquid-liquid extractions can only be an alternative when ion pairing is introduced. Ion pair extraction was used for the analysis of QA compounds in urine by Murray et al. after adjusting the $\mathrm{pH}$ of the urine to 10 and extracting the newly formed iodine-glycine drug complex into dichloromethane [27]. In this way, a simple ion-pair liquid/liquid extraction was obtained with recoveries ranging from $56 \%$ to $90 \%$ [9]. A similar ion-pair liquid-liquid extraction utilizing heptane sulfonate is used by Rudy et al. for the quantitation and confirmation of ipratropium in equine urine for screening purposes. Of course, many of the ion-pairing reagents, e.g., heptane sulfonate, do not lend themselves all too well to ESI-MS detection.
In our approach, ion-pairing liquid/liquid extraction with dichloromethane is adjusted to approximate the chemical environment of the ensuing LC-MS analysis by using the same ion-pairing agent as used for the chromatographic separation step. In this way HFBA acts as ion-pairing agent for both the extraction and chromatographic separation step. This report is a detailed presentation of the development, optimization and validation of this method for $p$ oriented quantitative measurements.

\section{Experimental}

\subsection{Chemicals}

Pure reference standard of glycopyrrolate (USP quality, GLY) was obtained from Boehringer Ingelheim Chemicals Inc. (Petersburg, USA). The internal standard, mepenzolate (Mp) was obtained as mepenzolate bromide from Sigma-Aldrich (Bornem, Belgium). LC-MS grade methanol and acetonitrile were from Biosolve (Valkenswaard, The Netherlands). Ultrapure water was produced by a Synergy 185 system (Millipore Comp., Bedford, MA, USA). Heptafluorobutyric acid, formic acid (p.a. 98\%-100\%), dichloromethane and ammonium formate were purchased at Sigma-Aldrich (Bornem, Belgium). Drug-free blank plasma (different patient pools) was obtained at the local blood bank and kept frozen until use.

\subsection{Analytical standards}

Individual stock solutions of GLY $\left(1.01 \times 10^{6} \mathrm{ng} / \mathrm{mL}\right)$ and $\mathrm{Mp}$ $\left(1.01 \times 10^{6} \mathrm{ng} / \mathrm{mL}\right)$ were prepared by accurately weighing the required amounts in separate volumetric flasks and dissolving in appropriate volumes of high purity water. These solutions were appropriately diluted in high purity water by serial dilution using a Hamilton diluter (Hamilton, Bonaduz, Switzerland) to obtain the working standard solutions used to spike plasma calibrators and quality control samples (see Section 2.3). The following standard solutions were prepared: $101 \times 10^{2} ; 504 \times 10^{1} ; 101 \times 10^{1}$; $504 \mathrm{ng} / \mathrm{mL} ; 101 ; 75.7$ and $50.4 \mathrm{ng} / \mathrm{mL}$. Separate weightings, dilutions and volumetric material were used in the preparation of the quality control samples which were also used in the accuracy investigation method. All standards were kept at $-20^{\circ} \mathrm{C}$ and were used for a maximum of 3 months.

\subsection{Calibrators and quality control samples}

Calibrators in plasma were prepared by adding $20 \mu \mathrm{L}$ of the relevant working standard solution to $1980 \mu \mathrm{L}$ of blank plasma. Calibrators over a concentration range of $0.1-100 \mathrm{ng} / \mathrm{mL}$ for GLY (101; $50.4 ; 10.1 ; 5.04 ; 1.01 ; 0.757 ; 0.504 ; 0.101 \mathrm{ng} / \mathrm{mL}$ ) were obtained. Quality control samples at three different levels $\left(\mathrm{QC}_{1} \quad 0.252, \mathrm{QC}_{2}\right.$ 2.52 , and $\mathrm{QC}_{3} 25.2 \mathrm{ng} / \mathrm{mL}$ ), different from the calibration concentrations, were separately prepared in larger pools.

Each calibration curve was acquired in triplicate allowing validation of the calibration curves based upon the Food and Drug Administration bioanalytical method validation guidance protocol [30]. In this context, precision, accuracy, limit of detection (LOD), lower and upper limit of quantification (LLOQ resp. ULOQ) and selectivity were also determined. To that end, calibration curves were run on 6 different days. The concurrently analyzed (different days, $n=6$ ), quality control samples were also used to calculate total precision and accuracy of the method. Using these samples, accuracy, as percentage error (100-[concentration(measuredadded)/added] $\times 100$ ), was assessed. Two of the quality control levels ( $\mathrm{QC}_{1} 0.252$ and $\mathrm{QC}_{3} 25.2 \mathrm{ng} / \mathrm{mL}$ ) were also used for a postextraction stability study. The samples were repeatedly re-analyzed 
over a $10 \mathrm{~h}$ time interval (time frame of 60 samples) and the resulting absolute peak areas were plotted against injection time. Evaluation of the slope of this curve together with the percentage response deviation compared to the time zero injection provides stability data.

\subsection{Sample preparation and extraction}

To $1 \mathrm{~mL}$ of plasma sample, a fixed amount of Mp, as internal standard, was added ( $50 \mu \mathrm{L}$ of a 1/2000 dilution of the stock solution; $25.2 \mathrm{ng}$ ). Subsequently, $1 \mathrm{~mL}$ of $0.2 \mathrm{M}$ ammonium formate (adjusted added. After $10 \mathrm{~min}$ of rotation on a rotatory mixer, the tubes were centrifuged for $20 \mathrm{~min}$ at $2254 \times \mathrm{g}\left(25^{\circ} \mathrm{C}\right) .750 \mu \mathrm{L}$ was removed from the upper water phase and transferred into new centrifuge tubes. After addition of $1 \mathrm{~mL}$ of $0.1 \mathrm{M}$ aqueous HFBA and $4 \mathrm{~mL}$ of dichloromethane, samples were rotated again on a rotatory mixer for $10 \mathrm{~min}$. After centrifugation ( $20 \mathrm{~min}, 2254 \times \mathrm{g}, 25^{\circ} \mathrm{C}$ ), the upper (water) phase was removed as waste. The lower dichloromethane layer was evaporated to dryness under a nitrogen stream at $35^{\circ} \mathrm{C}$ (TurboVap LV evaporator, Zymark). Following, dried samples were redissolved in $200 \mu \mathrm{L}$ of eluent A (15 mM HFBA-20 mM ammonium formate buffer) before injection.

\subsection{Mobile phases}

LC eluent A consisted of HFBA ( $15 \mathrm{mM}$ )-ammonium formate buffer $(20 \mathrm{mM})$ adjusted to $\mathrm{pH} 3.30$ with formic acid. Eluent $\mathrm{B}$ was pure methanol. Both solvents (A) and (B) were filtered over a $0.45 \mu \mathrm{m}$ membrane filter before use.

\subsection{Liquid chromatography}

The Atlantis dC18 (2.1 mm I.D., length $50 \mathrm{~mm}$, particle size $3 \mu \mathrm{m}$ ) used was purchased from Waters (Milford, MA, USA). The chromatographic system consisted of an Alliance 2795 LC system equipped with an analytical autosampler having a $100 \mu \mathrm{L}$ injection to $\mathrm{pH} 3.0$ with formic acid) and $4 \mathrm{~mL}$ of dichloromethane were

loop installed. Isocratic elution was performed with a fixed composition of $30 \%$ eluent $A$ and $70 \%$ eluent $B$ at a flow rate of $100 \mu \mathrm{L} / \mathrm{min}$. After injection of $20 \mu \mathrm{L}$ of sample, a total run cycle time of $10 \mathrm{~min}$ was used.

\subsection{Mass spectrometry}

The HPLC system was interfaced with a QTOF1 mass spectrometer (Micromass-Waters, Manchester, UK), equipped with an orthogonal electrospray source $\left(Z\right.$-spray $\left.{ }^{\circledR}\right)$ operated in the positive ion mode. The mass spectrometer was operated in the MS/MS mode allowing maximum sensitivity and specificity. Nitrogen acted both as nebulizer $(60 \mathrm{~L} / \mathrm{h})$ and drying gas $(580 \mathrm{~L} / \mathrm{h})$ and argon served as collision gas $\left(4.5 \times 10^{-3} \mathrm{mbar}\right.$ collision cell pressure). A standard $120 \mu \mathrm{m}$ capillary was used in the electrospray ionization (ESI) interface. The source was operated at $120^{\circ} \mathrm{C}$ and the desolvation temperature was $240^{\circ} \mathrm{C}$. Electrospray capillary and cone voltage were optimized to 3000 and $30 \mathrm{~V}$, respectively. Data were collected and processed using the MassLyn $x^{\circledR}$ and Quanlyn $x^{\circledR}$ software (Micromass-Waters, Manchester, UK). Collision energy was assessed to $30 \mathrm{eV}$ after infusion experiments with pure GLY (101 ng/mL, $m / z$ 318.4) and Mp standard (101 ng/mL, m/z 340.3) dissolved in the same eluent composition of the isocratic chromatographic system, at $10 \mu \mathrm{L} / \mathrm{min}$. Quantification, after collisioninduced dissociation of the respective precursor ions, $m / z 318.4$ and $\mathrm{m} / z 340.3$, occurs at $m / z 116.2$ and $m / z 130.2$ for GLY and Mp, respectively. In doing so, a sometimes so-called pseudo-MRM method is obtained. The MS/MS spectra and chemical structure of GLY and $\mathrm{Mp}$, as of their corresponding fragment ions are shown in Fig. 1.

\section{Results and discussion}

\subsection{Extraction procedure and its validation}

Using the proposed procedure, GLY is extracted using a combination of ion-pairing extraction with HFBA and liquid-liquid extraction. The same agent is also used as the mobile phase addi-
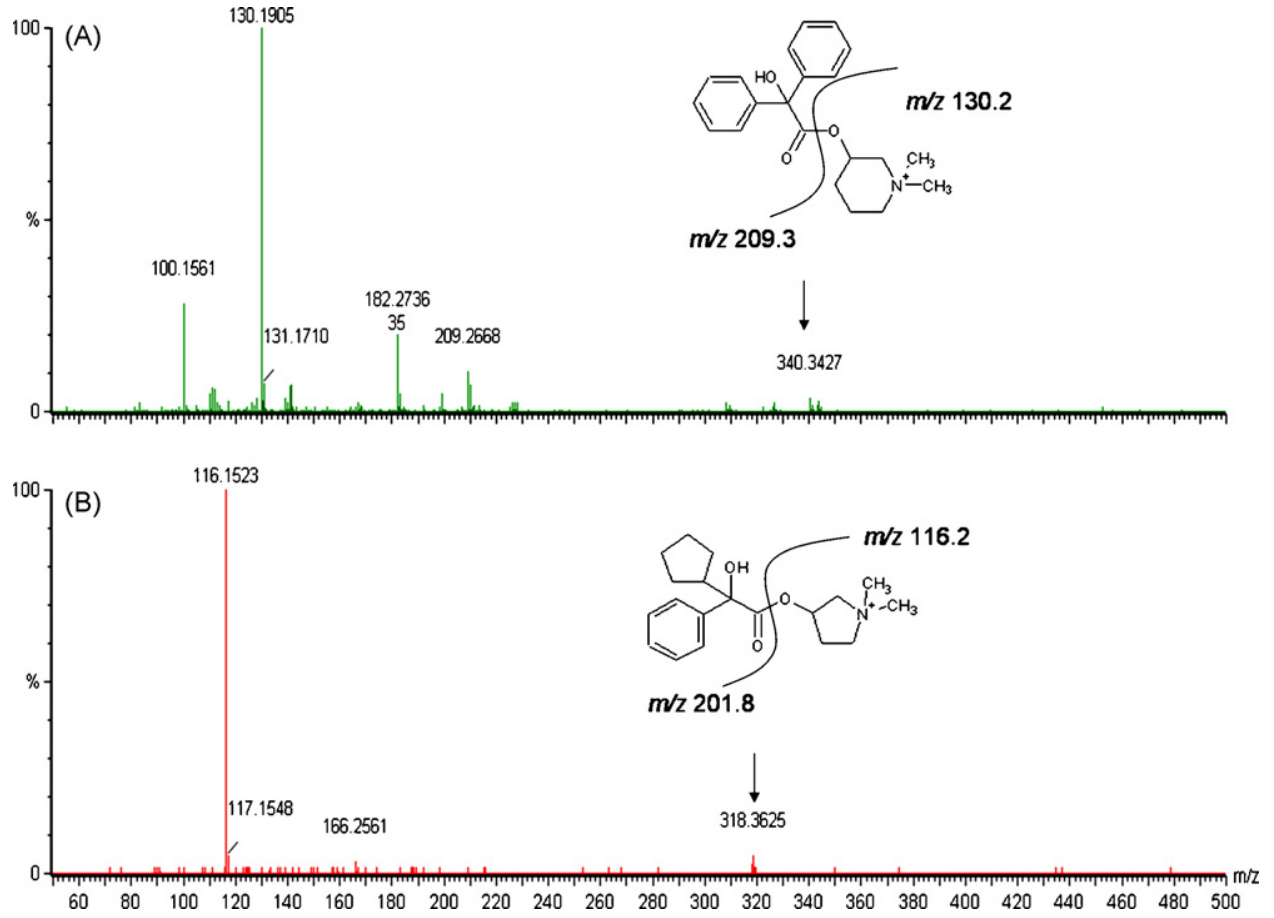

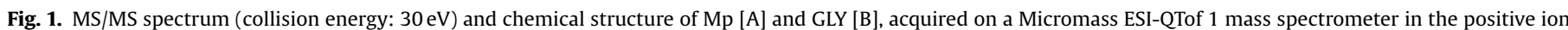
mode. $\mathrm{m} / \mathrm{z} 318.4$ (GLY) and $\mathrm{m} / \mathrm{z} 340.3$ were selected as precursor ion, $\mathrm{m} / \mathrm{z} 116.2$ (GLY) and $\mathrm{m} / \mathrm{z} 130.2$ (Mp) were selected for quantification. 
tive. In both cases, HFBA is added to counteract the highly polar characteristics of the quaternary ammonium group. During extraction, a broad range of proteins and lipids is removed in a first step under acidic conditions using dichloromethane. A major part of the upper water layer is separated from the dichloromethane layer by transferring $750 \mu \mathrm{L}$ to a new centrifugation tube. Nearly half of the water phase, composed of $1 \mathrm{~mL}$ of plasma sample and $1 \mathrm{~mL}$ of $0.2 \mathrm{M}$ ammonium formate is as such thrown away as waste. This proved inevitable, due to the formation of a white diffuse ring between the two phases, mainly made up of plasma proteins. Following, the HFBA solution is added and a second liquid-liquid extraction step with dichloromethane occurs. In contrast to the first step QA, like GLY and Mp, are now surrounded by the anionic counter-ions, becoming less polar as a whole and moving towards the dichloromethane phase. The total water layer is then thrown away and the resulting dichloromethane phase is now a highly pure extract of cationic substances like QA.

Thus, by the use of 2 consecutive liquid-liquid extraction steps, one with and one without the addition of the counter-ion HFBA to neutralize the permanent cationic nature of QA drugs, only minor plasma effects, a critical issue in the validation of the assay accuracy and reliability, are expected. Indeed, in bioanalytical mass spectrometry, absolute matrix effects are almost inescapable. However, much more important is the demonstrable lack of relative matrix effects, i.e., variance between patient results brought about by the inter-individual differences in the constitution of their plasma. Standard validation efforts, based on pooled blank biological fluids do not reveal this possibility peculiar to (ESI)-LC-MS. In fact, the assessment of matrix effect during development and validation of HPLC-MS(/MS) methods is also recommended by the U.S. Food and Drug Administration (FDA) "to ensure that precision, selectivity, and sensitivity will not be compromised" [30]. To that end, matrix (plasma) effects were evaluated based on the suggestions of Matuszewski et al. [31]. Thus, absolute matrix effect was assessed by spiking a quantity of target (GLY and Mp) into blank plasma extract and comparing the area to the same quantity spiked into the solvent. The same solvent composition $(30 / 70(\mathrm{v} / \mathrm{v})$ eluent $A /$ eluent $B$ ) as during the isocratic chromatographic separation step was used. This was done at all three of the QC concentration levels $(0.252 ; 2.52$ and $25.2 \mathrm{ng} / \mathrm{mL})$ and in three-fold. Moreover, the experiment was repeated for four plasma lots, originating from four different individuals, allowing the evaluation of relative matrix effects too. Using the aforementioned method, the matrix effect was $110 \% \pm 20.1$ (mean $\pm \mathrm{SD} ; n=3$ ) for $\mathrm{QC}_{1} ; 133 \% \pm 9.59 \%$ for $\mathrm{QC}_{2}$ and $120 \% \pm 6.84 \%$ for $\mathrm{QC}_{3}$. As can be seen, some ionization enhancement (matrix effect $>100 \%$ ) is present.

The assessment of the relative matrix effect was based on the variability of the peak area ratio GLY/Mp $(n=3)$ spiked into blank extracts of 4 different plasma lots [31]. In doing so, the relative matrix effect was $10.1 \% \pm 3.34$ (mean $\pm \mathrm{SD} ; n=4$ ) for $\mathrm{QC}_{1}$; $5.47 \% \pm 0.585$ for $\mathrm{QC}_{2}$ and $10.9 \% \pm 1.45$ for $\mathrm{QC}_{3}$. This variability seems to be comparable to or only slightly higher than the precision of determination of standards injected directly in the mobile phase $(9.64 \% \pm 0.776$ (mean $\pm S D ; n=3) ; 5.13 \% \pm 1.43$ and $6.72 \% \pm 0.491$, respectively). These data confirm that the relative matrix effect has practically no effect on the quantification of GLY.

The extraction efficiency was equally determined for the three levels of quality control samples, based on the recommendations by Matuszewski et al. as the concentration ratio of spiked plasma extract to blank plasma extract fortified with the same amount of compound [31]. In doing so, absolute extraction recovery was $41.8 \% \pm 2.22($ mean \pm SD; $n=3), 45.3 \% \pm 1.55$ and $42.1 \% \pm 4.27$ for $\mathrm{QC}_{1}, \mathrm{QC}_{2}$ and $\mathrm{QC}_{3}$. The latter extraction recoveries seem to be low. However, taking into account that approximately half of the water layer is discarded during the first liquid-liquid extraction step, potential extraction efficiency could be increased up to $80 \%$ by isolating the maximum volume possible of water layer. This, however, was not favored for ease of handling and in order to increase the extraction methods' reproducibility.

Process efficiency, describing both plasma effect and extraction efficiency combined, was calculated as the concentration ratio of spiked plasma extract to the same quantity spiked into pure eluent. For the 3 QC levels, process efficiency was $45.2 \% \pm 5.74$ (mean \pm SD; $n=3$ ); $56.9 \% \pm 6.41$ and $49.2 \pm 2.00$, respectively. Evidently, throwing away nearly half of the water layer during the first extraction step again affects these numbers.

\subsection{Chromatographic performance}

The chromatographic separation step takes 10 min under isocratic conditions, i.e., $70 \%$ of eluent B. Different isocratic eluent compositions were evaluated. $30: 70$ of $15 \mathrm{mM}$ HFBA in $20 \mathrm{mM}$ ammonium formate buffer ( $\mathrm{pH}$ 3.30): methanol was finally preferred as eluent composition based on retention time behavior. A minimal retention time ( $\mathrm{Tr}$ ) of $5 \mathrm{~min}$ was aimed at, allowing sufficient chromatographic separation of potentially co-extracted substances for one thing and a fast sample turnover time for another. In doing so, $\operatorname{Tr}$ was $6.82 \pm 0.140$ min (mean $\pm S D$; $n=36$ ) $\mathrm{min}$ for GLY and $6.02 \pm 0.152 \mathrm{~min}$ ( mean $\pm \mathrm{SD} ; n=36) \mathrm{min}$ for the IS Mp. Fig. 2 shows typical mass chromatograms of GLY and Mp for a blank serum, an LLOQ concentration level sample and a real patient sample (vide infra).

\subsection{Calibration and method validation}

For calibration purposes, the analyte to IS peak area ratio was plotted against analyte concentration and weighed regression analysis was applied to calculate the calibration curves. In doing so, data heteroscedasticity is counteracted and a higher weight is allocated to the lower concentrations in the calibration curve, leading to an improved quantification of the lower concentrations. A weighing factor $1 / \mathrm{X}$ was chosen, based on the analysis of residuals and accuracy of quality control samples. Quadratic calibration curves (eight calibration points, one zero sample) gave the best fit based on statistical regression analysis comparison (Statgraphics ${ }^{\circledR}$, Manugistics, Inc., Rockville, MD), the analysis of residuals (i.e., lowest absolute sum) and the acceptance criteria of the concomitant quality control samples $\left(\mathrm{QC}_{1} \quad 0.252, \mathrm{QC}_{2} 2.52\right.$, and $\left.\mathrm{QC}_{3} 25.2 \mathrm{ng} / \mathrm{mL}\right)$ calculated according to the different fits applied to a particular calibration set. The advent of powerful automated quantification software tools, e.g., Quanlynx ${ }^{\circledR}$, makes quadratic calibrations nearly as easy in use as linear ones, provided that one accepts the statistically enforced requirement to use a somewhat larger number of points in the calibration exercise ( 8 calibrators are used instead of a minimal of 6 required for linear calibration curves). Nowadays in LC-MS(/MS), especially with electrospray ionization (and in the field of TOF analyzers), linear calibration curves with a dynamic range exceeding 2 decades are hard to find. We have often seen that electrospray ionization does not generate analyte ions a priori by a linear process, especially not over a larger concentration area. The latter is especially true when analyzing biological extracts where extract impurity affects the ionization process. Thus, forcing a linear function through, in essence, curvilinear data rather introduces accuracy errors [32]. Moreover, quadratic calibration curves clearly provide an extended measurement interval, in our case from 0.101 to $101 \mathrm{ng} / \mathrm{mL}$. Indeed, a linear calibration correlation was only observed with the lower 6 calibrators included. At higher concentrations, the effectiveness of the electrospray ionization process begins to deviate from an absolute linear process (see Fig. 3). 
blanco -

070201 blextr1 $\quad$ 2: TOF MSMS ES+

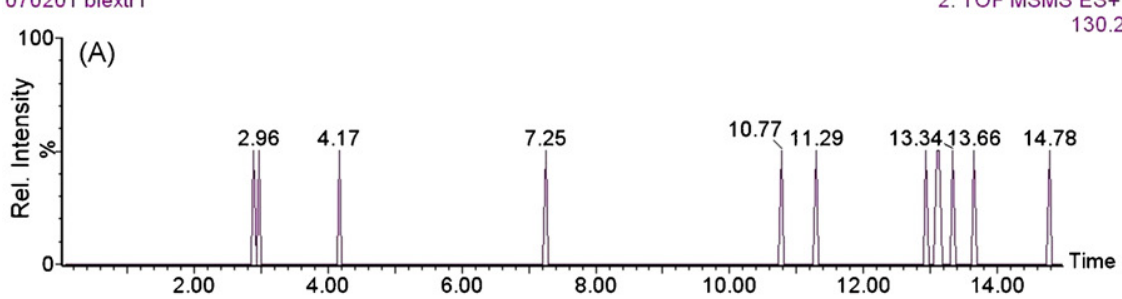

070201 blextr1

1: TOF MSMS ES+

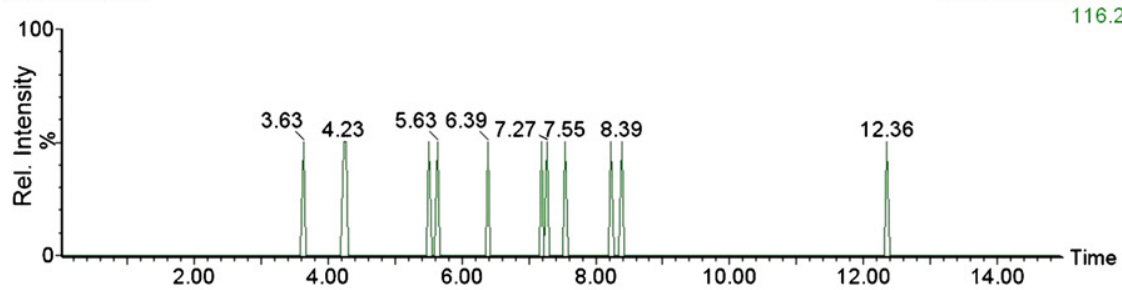

st1

070130 st1
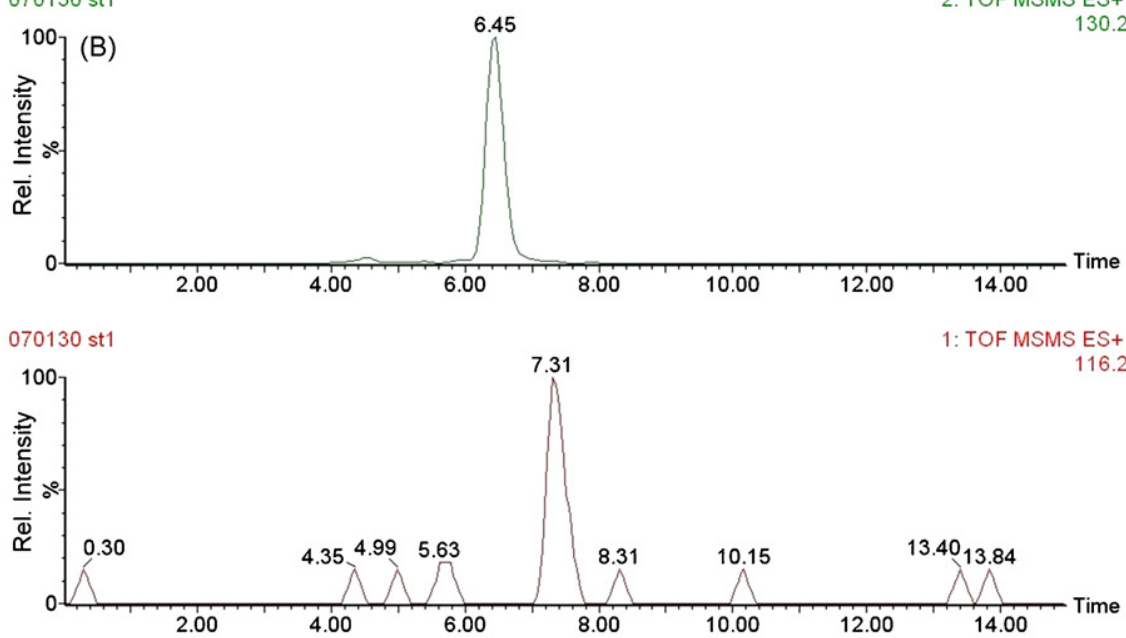

plasma T5 $\mathrm{nr} 570427$ 058A66/3001061114 $070201 \times 1$
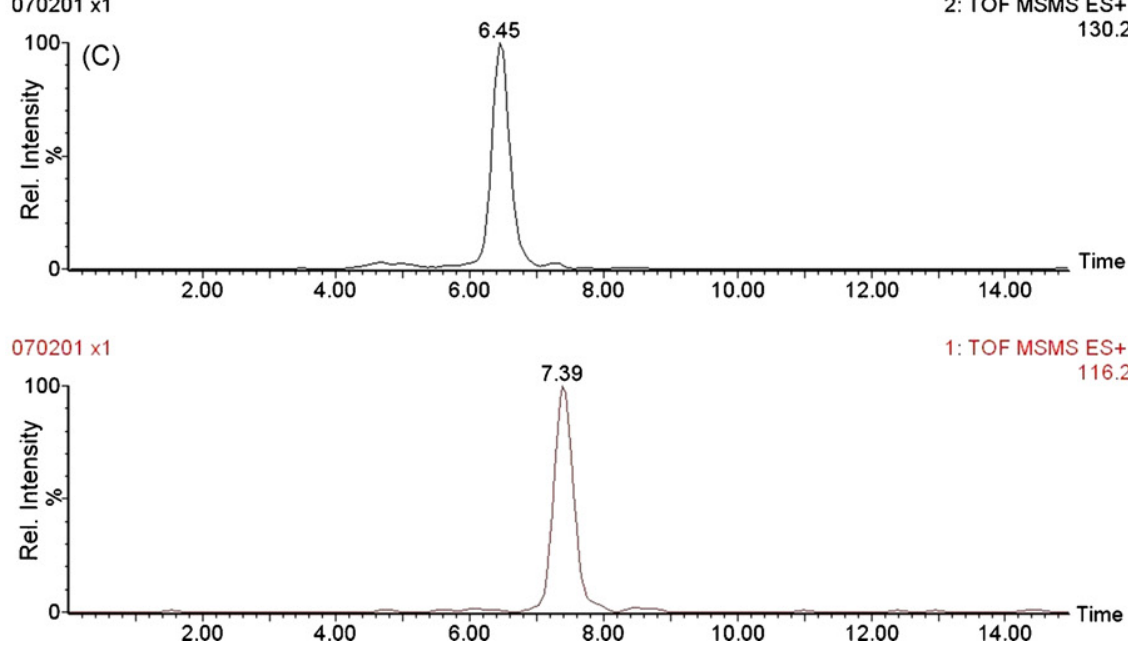

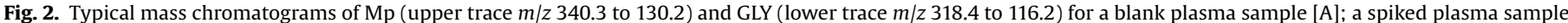
at the LLOQ concentration level [B]; and for a real patient sample [C]. 
Table 1

Accuracy, precision, absolute matrix effect, inter-subject variability, absolute recovery and process efficiency at the 3 QC concentration levels.

\begin{tabular}{|c|c|c|c|c|c|c|c|c|}
\hline & $\begin{array}{l}\text { Concentration } \\
(\mathrm{ng} / \mathrm{mL})\end{array}$ & $\begin{array}{l}\text { Accuracy } \\
(\% ; n=6)\end{array}$ & $\begin{array}{l}\text { Precision (total) } \\
(\% ; n=6)\end{array}$ & $\begin{array}{l}\text { Precision } \\
\text { (within-day) } \\
(\% ; n=4)\end{array}$ & $\begin{array}{l}\text { Absolute matrix effect } \\
(\text { mean } \% \pm S D ; n=3)\end{array}$ & $\begin{array}{l}\text { Inter-subject variability } \\
\text { (mean\% } \pm \mathrm{SD} ; n=4)\end{array}$ & $\begin{array}{l}\text { Absolute recovery } \\
(\text { mean } \% \pm \mathrm{SD} ; n=3)\end{array}$ & $\begin{array}{l}\text { Process efficiency } \\
(\text { mean } \% \pm \mathrm{SD} ; \\
n=3 \text { ) }\end{array}$ \\
\hline LLOQ & 0.101 & 88.9 & 18.0 & - & - & - & - & - \\
\hline $\mathrm{QC}_{1}$ & 0.252 & 112 & 13.9 & 7.22 & $111 \pm 20.1$ & $10.1 \pm 3.34$ & $41.8 \pm 2.22$ & $45.2 \pm 5.74$ \\
\hline $\mathrm{QC}_{2}$ & 2.52 & 105 & 9.32 & 8.43 & $133 \pm 9.59$ & $5.47 \pm 0.585$ & $45.3 \pm 5.55$ & $56.9 \pm 6.41$ \\
\hline $\mathrm{QC}_{3}$ & 25.2 & 88.1 & 8.63 & 7.50 & $120 \pm 6.84$ & $10.9 \pm 1.45$ & $42.1 \pm 4.27$ & $49.2 \pm 2.00$ \\
\hline
\end{tabular}

The mean values $( \pm \mathrm{SD}, n=6)$ of the quadratic equation coefficients were: $y=-2.21 \times 10^{-4}\left( \pm 3.93 \times 10^{-5}\right) \times x^{2}+5.85 \times 10^{-2}$ $\left( \pm 5.27 \times 10^{-3}\right) \times x+4.08 \times 10^{-3}\left( \pm 4.82 \times 10^{-4}\right)$. The mean coefficient of determination $\left(R^{2}\right)$ was 0.9995 ( $n=6$, RSD\% 0.0216). ANOVA analysis always revealed a statistically significant calibration relationship at the $99 \%$ confidence level. The $95 \%$ confidence interval for the intercept always included zero, statistically indicating that the calibration curve passes through zero. The lower limit of quantification (LLOQ) was established at the level of the lowest calibrator, i.e., $0.101 \mathrm{ng} / \mathrm{mL}$, provided that an acceptable level of precision and accuracy is achieved. At this concentration, a signal to noise ratio of 5.2 , total precision (RSD\%, $n=6$ ) of $18.0 \%$ and accuracy $(n=6)$ of $88.9 \%$ was noted. These data are within the generally accepted validation criteria limits (precision $<20 \%$ and accuracy between 80 and $120 \%$ ). Accordingly, the ULOQ was established at the highest calibrator, being $101 \mathrm{ng} / \mathrm{mL}$. The, less informative, limit of detection (LOD) was defined at a signal to noise ratio equal to 3 and approximated from the lowest calibrator. In doing so, an LOD for GLY of nearly $0.050 \mathrm{ng} / \mathrm{mL}$ is achieved. Accuracy and precision (within-day or repeatability and total precision) of the LC-MS(/MS) method were further evaluated using the 3 different quality control levels. Within-day precision or repeatability remained below $9 \%$, total precision below $14.0 \%$. Inaccuracy was at every QC concentration level below 15\%. Again, these data are well within the generally required validation criteria limits, being maximum inaccuracy of $15 \%$ (20\% at LLOQ) and precision below 15\% (20\% at LLOQ). All of the abovementioned validation parameters are summarized in Table 1. Post-extraction stability was evaluated at the $\mathrm{QC}_{1}$ and $\mathrm{QC}_{3}$ concentration levels. A plot of the resulting absolute peak areas against injection time revealed a small positive slope for the $\mathrm{QC}_{1}$ concentration and a small negative slope for the $\mathrm{QC}_{3}$ concentration. However, the percentage deviation compared to the time zero injection was less than $13 \%\left(\mathrm{QC}_{1}\right)$, respectively $14 \%\left(\mathrm{QC}_{3}\right)$ indicating acceptable stability.

Selectivity is an inherent aspect of LC-MS/MS procedures and was confirmed by the absence of signal in at least 6 blank plasma extracts of different patient origins.

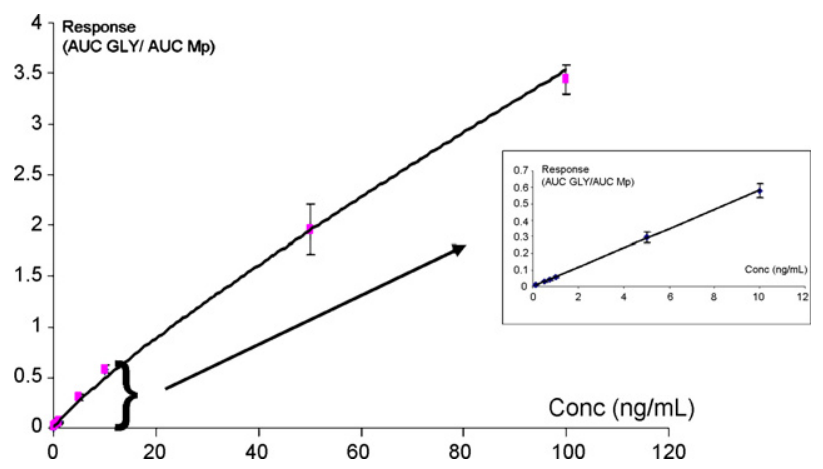

Fig. 3. Curvilinear calibration function of GLY, ranging from 0.101 to $101 \mathrm{ng} / \mathrm{mL}$. When the 2 highest calibrators ( 101 and $50.4 \mathrm{ng} / \mathrm{mL}$ ) are not included in the concentration response curve, the latter shifts to a more linear function.

\subsection{Study samples}

The development and validation of the analytical method was performed as part of a clinical study for the pharmacokinetic evaluation of GLY concentrations after a single intravenous bolus injection of Robinul ${ }^{\circledR}$. Patient samples are sent to us by the Ghent University Hospital (Department of Anesthesia). Informed consent is obtained from all participants of the study, refusal to sign an informed consent means exclusion from the study. In a preliminary proof-of-concept trial, one female patient was selected (height: $159 \mathrm{~cm}$; weight: $73 \mathrm{~kg}$ ). The patient had been treated preoperatively with tamoxifen for 7 days. Before the operation, one bolus injection of Robinul ${ }^{\circledR}$, containing $0.4 \mathrm{mg}$ GLY, had been given and a blood sample was taken (T0). Following, a second and third sample were taken after 5 and 10 min (T5 and T10), respectively. All the samples were collected through an arterial line, plasma was separated from the blood and stored at $-80^{\circ} \mathrm{C}$. The study samples were analyzed in batch in a sequence consisting of 2 separate calibration sample sets with the study samples in between and the required quality control samples interspersed within the sequence. In doing so, the measured concentration of GLY was 12.9 and $4.93 \mathrm{ng} / \mathrm{mL}$ for T5 (see chromatogram in Fig. 2C) and T10, respectively. For T0, no GLY concentration was measurable nor detectable. The actual study will allow, among other clinical study objectives, full evaluation of the pharmacokinetic characteristics of GLY after a single bolus injection with Robinul ${ }^{\circledR}$ based on an extended number of study patients. This study phase is in a preliminary stage.

\section{Conclusion}

A fully validated LC-MS(/MS) method was developed for the determination of GLY in human plasma. Method evaluation was performed in accordance to the widely accepted international validation standards as proposed by the US FDA. To obtain a reliable LC-MS procedure, adequate sample clean-up proved necessary. This was achieved by a simple and relatively fast two step liquid-liquid extraction procedure, a part with and without the use of the counter-ion HFBA to neutralize the permanently positive charge state of $\mathrm{QA}$ drugs. In doing so, during validation relative and absolute matrix effects were found to be of insignificant influence on the quantitation procedure. Other mandatory validation results were also in concordance with internationally accepted performance criteria. The same counter-ion was also used during the chromatographic separation step using a standard C18 phase. The isocratic eluent LC-MS(/MS) method takes only 10 min allowing the quantification of GLY over a concentration range of 3 decades with a fast sample turnover time.

\section{Acknowledgements}

This work was supported by grant B/06859-BOF06/24j/025 (Bijzonder OnderzoeksFonds of the Ghent University) and grant G.0320.02 (FWO-Vlaanderen). 


\section{References}

[1] A. Clifford, C.A. Bernstein, J.H. Waters, M.C. Torjman, D. Ritter, J. Clin. Anesth. 8 (1996) 515.

[2] L. Manchikanti, J.R. Roush, Anesth. Analg. 63 (1984) 40.

[3] D.M. Dewan, A.S. Wheeler, F.M. James, H.M. Floyd, L. Rhyne, Can. Anaesth. Soc. J. 29 (1982) 27.

[4] R.K. Mirakhur, J. Reid, J. Elliott, Anaesthesia 34 (1979) 453.

[5] T. Ali-Melkkila, T. Kaila, J. Kanto, Acta Anaesthesiol. Scand. 33 (1989) 513.

[6] J. Penttilä, A. Helminen, K. Luomala, H. Scheinin, Eur. J. Clin. Pharmacol. 57 (2001) 153.

[7] L.C. Matassa, D. Woodard, R.K. Leavitt, P. Firby, P. Baumier, J. Chromatgr. 573 (1992) 43.

[8] R. Leavitt, P. Firby, W. Farmer, P. Paterson, Proceedings on the 44th Annual Meeting of the Association of Official Racing Chemists, Cincinnatti, OH, May, 1991.

[9] F.P.W. Tang, G.N. Leung, T.S.M. Wan, Electrophoresis 22 (2001) 2201.

[10] A.M. Duffield, P.J. Reilly, D. Nelson, H. Dama, C.J. Suann, Proc. 44th AORC, Cincinnatti, OH, May, 1991.

[11] Mui, L. Downey, D.E. Auer, R. Houghton, Proc. 11th Int. Conf. Racing Anal. Vet., Queensland, 448-193, 1996.

[12] R. Castro, E. Moyano, M.T. Galceran, J. Chromatogr. A 830 (1999) 145.

[13] M.C. Carneiro, L. Puignou, M.T. Galceran, J. Chromatogr. A 669 (1994) 217.

[14] M.T. Galceran, M.C. Carneiro, M. Diez, L. Puignou, J. Chromatogr. A 782 (1997) 289.
[15] D. Kaniansky, F. Ivnyi, F.I. Onuska, Anal. Chem. 66 (1994) 1817.

[16] D. Barceló, G. Durand, R.J. Vreeken, J. Chromatogr. 647 (1993) 271.

[17] T. Itagaki, S.J. Lai, S.R. Binder, J. Liq. Chromatogr. Rel. Technol. 20 (1997) 3339.

[18] R. Kesari, M. Rai, V.K. Gupta, J. Assoc. Off. Anal. Chem. 80 (1997) 388.

[19] C. Fuke, K. Ameno, S. Ameno, T. Kiriu, T. Shinohara, K. Sog, I. Ijirin, J. Anal. Toxicol. 16 (1992) 214.

[20] G.J. Moody, R.K. Owusu, J.D. Thomas, Anal. Lett. 21 (1988) 1653.

[21] K. Watabe, K. Okada, T. Katsu, J. Toxicol. Environ. Health 38 (1992) 142.

[22] K. Ameno, C. Fuke, S. Ameno, H. KInoshita, I. Ijiri, J. Liq. Chromatogr. 18 (1995) 2115.

[23] T.M. Chichila, S.M. Walters, J. Assoc. Off. Anal. Chem. 74 (1991) 961.

[24] B.L. Worobey, J. Assoc. Off. Anal. Chem. 76 (1993) 881.

[25] J. Hajslová, P. Cuhra, T. Davídek, K. Davídek, J. Chromatogr. 479 (1989) 243.

[26] R.J. Vreeken, W.D. Vandongen, R.T. Ghijsen, G.J. Dejong, H. Lingeman, U.A.T Brinkman, R.G.J. Vanleuken, G.T.C. Kwakkenbos, R.S. Deelder, Biol. Mass Spectrom. 21 (1992) 305.

[27] G.R. Murray, T.N. Calvey, N.E. Williams, K. Chan, J. Chromatogr. 308 (1984) 143

[28] M. Ariffin, R.A. Anderson, J. Chromatogr. B 842 (2006) 91.

[29] Y. Pico, G. Font, J.C. Moltó, J. Mañes, J. Chromatogr. A 885 (2000) 251.

[30] Guidance for Industry. Bioanalytical Method Validation. U.S. Department of Health and Human Services, Food and Drug Administration. May 2001.

[31] B.K. Matuszewski, M.L. Constanzer, C.M. Chavez-Eng, Anal. Chem. 75 (2003) 3019.

[32] M.L. Storme, F.H. Jansen, W. Goeteyn, J.F. Van Bocxlaer, Rapid Commun. Mass Spectrom. 19 (2006) 2947. 